Proyecciones Journal of Mathematics

Vol. 37, No 3, pp. 547-564, September 2018.

Universidad Católica del Norte

Antofagasta - Chile

\title{
Some new triple sequence spaces over $n$-normed space
}

\author{
Tanweer Jalal \\ National Institute of Technology, India \\ and \\ Ishfaq Ahmad Malik \\ National Institute of Technology, India \\ Received : November 2017. Accepted : April 2018
}

\begin{abstract}
Triple sequence spaces were introduced by Sahiner et al. [27, 28]. The main objective of this paper is to define some new classes of triple sequences over $n$-normed space by means of Museiak-Orlicz function and difference operators. We also study some algebraic and topological properties of these new sequence spaces.
\end{abstract}

2010 Mathematics Subject Classification : 40C05, 46A45, $46 E 30$.

Keywords : Triple sequence spaces, Paranormed spaces, $n$-normed spaces, Musielak-Orlicz function. 


\section{Introduction}

By $w^{\prime \prime \prime}$ we shall denote the class of all complex triple sequence $\left\langle a_{i j k}\right\rangle$, where $i, j, k \in \mathbf{N}$, the set of positive integers. Then, $w^{\prime \prime \prime}$ is a linear space under the coordinate wise addition and scalar multiplication.

A triple sequence can be represented by a matrix, in case of double sequences we write in the form of a square. In case of triple sequence it will be in the form of a box in three dimensions. The different types of notions of triple sequence was introduced and investigated initially by Sahiner et al. [27, 28], Esi [7], Esi and Catalbas [8], Esi and Savas [9], Datta [2], Debnath [3] and many others.

The concept of 2-normed spaces was initially developed by Gähler [11], in the mid of 1960's while that of $n$-normed spaces was studied by Misiak [21]. Since then many authors have studied $n$-normed spaces and obtained various results, see Gunawan $([12,13])$ and Gunawan and Mashadi [14].

Kizmaz [19] introduced the notion of difference sequence spaces as follows for$$
Z(\Delta)=\left\{x=\left(x_{k}\right) \in w:\left(\Delta x_{k}\right) \in Z\right\}
$$

$Z=\ell_{\infty}, c$ and $c_{0}, \Delta(x)=x_{k}-x_{k+1}, \Delta^{0}(x)=x_{k}$.

The study was further generalized by Et and Colak [10] by introducing the spaces $\ell_{\infty}\left(\Delta^{n}\right), c\left(\Delta^{n}\right)$ and $c_{0}\left(\Delta^{n}\right)$.

The difference operator on triple sequence is defined as

$$
\begin{aligned}
& \Delta x_{m n k}=x_{m n k}-x_{(m+1) n k} \\
& -x_{m(n+1) k}-x_{m n(k+1)}+x_{(m+1)(n+1) k} \\
& +x_{(m+1) n(k+1)}+x_{m(n+1)(k+1)}-x_{(m+1)(n+1)(k+1)} .
\end{aligned}
$$

\section{Definitions and preliminaries}

Definition 2.1. [27] A triple sequence $\left\langle a_{i j k}\right\rangle$ is said to be convergent to $L$ in Pringsheim's sense if for every $\epsilon>0$, there exists $N(\epsilon) \in \mathbf{N}$ such that

$$
\left|a_{i j k}-L\right|<\epsilon \quad \text { whenever } \quad i \geq N, j \geq N, k \geq N
$$

and is written as $\lim _{i, j, k \rightarrow \infty} a_{i j k}=L$. 
Note: A triple sequence is convergent in Pringsheim's sense may not be bounded [27].

Definition 2.2. [27] A triple sequence $\left\langle a_{i j k}\right\rangle$ is said to be Cauchy sequence if for every $\epsilon>0$, there exists $N(\epsilon) \in \mathbf{N}$ such that

$$
\left|a_{i j k}-a_{l m n}\right|<\epsilon \quad \text { whenever } \quad i \geq l \geq N, j \geq m \geq N, k \geq n \geq N
$$

Definition 2.3. [27] A triple sequence $\left\langle a_{i j k}\right\rangle$ is said to be bounded if there exists $M>0$, such that $\left|a_{i j k}\right|<M$ for all $i, j, k \in \mathbf{N}$.

Definition 2.4. [2]A Triple sequence space $Y$ is said to be Solid if $\left\langle\alpha_{i j k} a_{i j k}\right\rangle \in$ $Y$ whenever $\left\langle a_{i j k}\right\rangle \in Y$ and for all triple sequences $\left\langle\alpha_{i j k}\right\rangle$ of scalars with $\left|\alpha_{i j k}\right| \leq 1$, for all $i, j, k \in \mathbf{N}$.

Definition 2.5. [2] A Triple sequence space $Y$ is said to be monotone if it contains the canonical pre-images of all its step spaces .

Note: A sequence space is solid implies that it is monotone.

Definition 2.6. [18],[24]

(Orlicz function and Musielak-Orlicz function)

An Orlicz function is a function $M:[0, \infty) \rightarrow[0, \infty)$ which is continuous, non-decreasing and convex with $M(0)=0, M(x)>0$, for $x>0$ and $M(x) \rightarrow \infty$ as $x \rightarrow \infty$. If convexity of Orlicz function $M$ is replaced by $M(x+y) \leq M(x)+M(y)$; then this function is called modulus function.

Lindenstrauss and Tzafriri [20] used the idea of Orlicz function to construct Orlicz sequence space:

$$
\ell_{M}=\left\{x \in \omega: \sum_{k=1}^{\infty} M\left(\frac{\left|x_{k}\right|}{\rho}\right)<\infty, \quad \text { for some } \rho>0\right\}
$$


The space $\ell_{M}$ with the norm:

$$
\|x\|=\inf \left\{\rho>0: \sum_{k=1}^{\infty} M\left(\frac{\left|x_{k}\right|}{\rho}\right) \leq 1\right\}
$$

becomes a Banach space which is called an Orlicz sequence space. For $M(t)=t^{p} \quad(1 \leq p<\infty)$, the space $\ell_{M}$ coincide with the classical sequence space $\ell_{p}(p \geq 1)$.

A sequence $f=\left(f_{m n k}\right)$ of Orlicz function is called a Musielak-Orlicz function. A sequence $g=\left(g_{m n k}\right)$ defined by

$$
g_{m n k}(v)=\sup \left\{|v| u-\left(f_{m n k}\right)(u): u \geq 0\right\} \quad m, n, k=1,2,3, \cdots
$$

is called the complementary function of a Musielak-Orlicz function $f$. For a given Musielak-Orlicz function $f$, the Musielak-Orlicz sequence space $t_{f}$ is defined as follows:

$$
t_{f}=\left\{x \in w^{\prime \prime \prime}: I_{f}\left(\left|x_{m n k}\right|\right)^{\frac{1}{m+n+k}} \rightarrow 0 \quad \text { as } m, n, k \rightarrow \infty\right\}
$$

where $I_{f}$ is a convex modulus function defined by:

$$
I_{f}(x)=\sum_{m=1}^{\infty} \sum_{n=1}^{\infty} \sum_{k=1}^{\infty} f_{m n k}\left(\left|x_{m n k}\right|\right)^{\frac{1}{m+n+k}}, \quad x=\left(x_{m n k}\right) \in t_{f} .
$$

Orlicz function $M$ satisfies $\Delta_{2}$-condition if and only if for any constant $L>1$ there exists a constant $K(L)$ such that $M(L u) \leq K(L) M(u)$ for all values of $u \geq 0$. An Orlicz function $M$ can always be represented in the following integral form

$$
M(x)=\int_{0}^{x} \eta(t) d t
$$

Where $\eta$ is known as the kernel of $M$, is right differentiable for $t \geq 0$, $\eta(0)=0, \eta(t)>0, \quad \eta$ is non-decreasing and $\eta(t) \rightarrow \infty$ as $t \rightarrow \infty$.

Definition 2.7 ( $n$-Normed Space). Let $n \in \mathbf{N}$ and $X$ be a linear space over the field $\mathbf{R}$ of reals of dimension $d$, where $2 \leq d \leq n$. A real valued function $\|\cdot, \ldots, \cdot\|$ on $X^{n}$ satisfying the following four conditions:

(1) $\left\|x_{1}, x_{2}, \ldots, x_{n}\right\|=0$ if and only if $x_{1}, x_{2}, \ldots, x_{n}$ are linearly dependent in $X$; 
(2) $\left\|x_{1}, x_{2}, \ldots, x_{n}\right\|$ is invariant under permutation;

(3) $\left\|\alpha x_{1}, x_{2}, \ldots, x_{n}\right\|=|\alpha|\left\|x_{1}, x_{2}, \ldots, x_{n}\right\|$ for any $\alpha \in \mathbf{R}$;

(4) $\left\|x_{1}+x_{1}^{\prime}, x_{2}, \ldots, x_{n}\right\| \leq\left\|x_{1}, x_{2}, \ldots, x_{n}\right\|+\left\|x_{1}^{\prime}, x_{2}, \ldots, x_{n}\right\|$;

is called an $n$-norm on $X$ and $(X,\|\cdot, \ldots, \cdot\|)$ is called an $n$-normed space over the field $\mathbf{R}$.

For example $\left(\mathbf{R}^{n},\|\cdot, \ldots, \cdot\|_{E}\right)$ where

$$
\begin{aligned}
& \left\|x_{1}, x_{2}, \ldots, x_{n}\right\|_{E}=\text { the volume of the } \\
& n-\text { dimensional parallelopiped spanned by the vectors } \\
& x_{1}, x_{2}, \ldots, x_{n}
\end{aligned}
$$

which can also be written as

$$
\left\|x_{1}, x_{2}, \ldots, x_{n}\right\|_{E}=\left|\operatorname{det}\left(x_{i j}\right)\right|
$$

where $x_{i}=\left(x_{i 1}, x_{i 2}, \cdots, x_{i n}\right) \in \mathbf{R}^{n}$ for each $i=1,2, \cdots, n$. Let $(X,\|\cdot, \ldots, \cdot\|)$ be an $n$-normed space of dimension $2 \leq n \leq d$ and $\left\{a_{1}, a_{2}, \cdots, a_{n}\right\}$ be linearly independent set in $X$. Then the following function $\|\cdot, \ldots, \cdot\|_{\infty}$ on $X^{n-1}$ defined by

$$
\left\|x_{1}, x_{2}, \ldots, x_{n-1}\right\|_{\infty}=\max \left\{\left\|x_{1}, x_{2}, \ldots, x_{n-1}, a_{i}\right\|: i=1,2, \ldots, n\right\}
$$

defines an $(n-1)$-norm on $X$ with respect to $\left\{a_{1}, a_{2}, \ldots, a_{n}\right\}$.

A sequence $\left(x_{k}\right)$ in a $n$-normed space $(X,\|\cdot, \ldots, \cdot\|)$ is said to converge to some $L \in X$ if

$$
\lim _{k \rightarrow \infty}\left\|x_{k}-L, z_{1}, \ldots, z_{n-1}\right\|=0 \quad \text { for every } \quad z_{1}, \ldots, z_{n-1} \in X
$$

A sequence $\left(x_{k}\right)$ in a $n$-normed space $(X,\|\cdot, \ldots, \cdot\|)$ is said to be Cauchy if

$$
\lim _{k, p \rightarrow \infty}\left\|x_{k}-x_{p}, z_{1}, \ldots, z_{n-1}\right\|=0 \quad \text { for every } \quad z_{1}, \ldots, z_{n-1} \in X .
$$

If every Cauchy sequence in $X$ converges to some $L \in X$, then $X$ is said to be complete with respect to the $n$-norm. Any complete $n$-normed space is said to be $n$-Banach space. The n-normed space has been studied in stretch ([1], [4], [5], [6], [21], [22],[23]). 


\section{Definition 2.8. [30] (Paranormed Space)}

Let $X$ be a linear metric space. A function $p: X \rightarrow \mathbf{R}$ is called paranorm, if

(1) $p(x) \geq 0$, for all $x \in X$

(2) $p(-x)=p(x)$, for all $x \in X$

(3) $p(x+y) \leq p(x)+p(y)$, for all $x, y \in X$

(4) If $\left(\lambda_{n}\right)$ is a sequence of scalars with $\lambda_{n} \rightarrow \lambda$ as $n \rightarrow \infty$ and $\left(x_{n}\right)$ is a sequence of vectors with $p\left(x_{n}-x\right) \rightarrow 0$ as $n \rightarrow \infty$, then $p\left(\lambda_{n} x_{n}-\right.$ $\lambda x) \rightarrow 0$ as $n \rightarrow \infty$.

A paranorm $p$ for which $p(x)=0$ implies $x=0$ is called total paranorm and the pair $(X, p)$ is called a total paranormed space. It is well known that the metric of any linear metric space is given by some total paranorm. For further reference on paranormed space ([15-17], [25] , [26]).

$$
\text { If } p=\left(p_{i j k}\right) \text { is a triple sequence of positive real numbers with }
$$

$$
0 \leq p_{i j k} \leq \sup p_{i j k}=G, K=\max \left(1,2^{G-1}\right)
$$

Then

$$
\left|a_{i j k}+b_{i j k}\right|^{p_{i j k}} \leq K\left\{\left|a_{i j k}\right|^{p_{i j k}}+\left|b_{i j k}\right|^{p_{i j k}}\right\}
$$

for all $i, j, k$ and triple sequences $a_{i j k}, b_{i j k} \in \mathbf{C}$. Also $|a|^{p_{i j k}} \leq \max \left(1,|a|^{G}\right)$ for all $a \in \mathbf{C}$.

\section{Construction of triple $n$-normed sequence spaces}

Now we introduce the new class of triple sequence spaces using Orlicz functions and difference operator on $n$ - normed space, if $M$ is an Orlicz function and $p=\left\langle p_{i j k}\right\rangle$ is a triple sequence of strictly positive real numbers and $(X,\|\cdot, \ldots, \cdot\|)$ is a real linear $n$-normed space we define the following classes of sequences: 


$$
\begin{aligned}
& W^{\prime \prime \prime}(M, \Delta, p,\|\cdot, \ldots, \cdot\|)=\left\{\left\langle a_{i j k}\right\rangle \in w^{\prime \prime \prime}: \lim _{l, m, n} \frac{1}{\frac{1}{\operatorname{lm}_{i j k}}}\right. \\
& \sum_{i=1}^{l} \sum_{j=1}^{m} \sum_{k=1}^{n}\left(M\left(\left\|\frac{\Delta a_{i j k}-L}{\rho}, z_{1}, \cdots, z_{n-1}\right\|\right)\right)^{p_{i j k}}=0,
\end{aligned}
$$

for each $z_{1}, \cdots, z_{n-1} \in X$, for some $\rho>0$ and $\left.L>0\right\}$,

$W_{0}^{\prime \prime \prime}(M, \Delta, p,\|\cdot, \ldots, \cdot\|)=\left\{\left\langle a_{i j k}\right\rangle \in w^{\prime \prime \prime}: \lim _{l, m, n} \frac{1}{l m n}\right.$

$\sum_{i=1}^{l} \sum_{j=1}^{m} \sum_{k=1}^{n}\left(M\left(\left\|\frac{\Delta a_{i j k}}{\rho}, z_{1}, \cdots, z_{n-1}\right\|\right)\right)^{p_{i j k}=}=0$,

for each $z_{1}, \cdots, z_{n-1} \in X$, for some $\left.\rho>0\right\}$, and

$$
\begin{aligned}
& W_{\infty}^{\prime \prime \prime}(M, \Delta, p,\|\cdot, \ldots, \cdot\|) \\
& =\left\{\left\langle a_{i j k}\right\rangle \in w^{\prime \prime \prime}: \sup _{l, m, n} z_{1}, \cdots, z_{n-1} \in X\right. \\
& \frac{1}{l m n} \sum_{i=1}^{l} \sum_{j=1}^{m} \sum_{k=1}^{n} \\
& \left(M\left(\left\|\frac{\Delta a_{i j k}}{\rho}, z_{1}, \cdots, z_{n-1}\right\|\right)\right)^{p_{i j k}}<\infty,
\end{aligned}
$$

for some $\rho>0\}$,

\section{Some Special Cases}

(i). If we take $p=\left(p_{i j k}\right)=1$, we get

$$
\begin{aligned}
& W^{\prime \prime \prime}(M, \Delta,\|\cdot, \ldots, \cdot\|)=\left\{\left\langle a_{i j k}\right\rangle \in w^{\prime \prime \prime}: \lim _{l, m, n} \frac{1}{l m n}\right. \\
& \sum_{i=1}^{l} \sum_{j=1}^{m} \sum_{k=1}^{n}\left(M\left(\left\|\frac{\Delta a_{i j k}-L}{\rho}, z_{1}, \cdots, z_{n-1}\right\|\right)\right)=0,
\end{aligned}
$$

for each $z_{1}, \cdots, z_{n-1} \in X$, for some $\rho>0$ and $\left.L>0\right\}$, $W_{0}^{\prime \prime \prime}(M, \Delta,\|\cdot, \ldots, \cdot\|)=\left\{\left\langle a_{i j k}\right\rangle \in w^{\prime \prime \prime}: \lim _{l, m, n} \frac{1}{l m n}\right.$

$$
\sum_{i=1}^{l} \sum_{j=1}^{m} \sum_{k=1}^{n}\left(M\left(\left\|\frac{\Delta a_{i j k}}{\rho}, z_{1}, \cdots, z_{n-1}\right\|\right)\right)=0,
$$

for each $z_{1}, \cdots, z_{n-1} \in X$, for some $\left.\rho>0\right\}$, and

$$
\begin{aligned}
& W_{\infty}^{\prime \prime \prime}(M, \Delta,\|\cdot, \ldots, \cdot\|) \\
& =\left\{\left\langle a_{i j k}\right\rangle \in w^{\prime \prime \prime}: \sup _{l, m, n} z_{1}, \cdots, z_{n-1} \in X \frac{1}{l m n} \sum_{i=1}^{l} \sum_{j=1}^{m} \sum_{k=1}^{n}\right. \\
& \left(M\left(\left\|\frac{\Delta a_{i j k}}{\rho}, z_{1}, \cdots, z_{n-1}\right\|\right)\right)<\infty,
\end{aligned}
$$

for some $\rho>0\}$,

(ii). If we take $M(x)=x$, we get 
$W^{\prime \prime \prime}(\Delta, p,\|\cdot, \ldots, \cdot\|)=\left\{\left\langle a_{i j k}\right\rangle \in w^{\prime \prime \prime}:\right.$

$\lim _{l, m, n} \frac{1}{l m n} \sum_{i=1}^{l} \sum_{j=1}^{m} \sum_{k=1}^{n}\left(\left\|\frac{\Delta a_{i j k}-L}{\rho}, z_{1}, \cdots, z_{n-1}\right\|\right)^{p_{i j k}}=0$,

for each $z_{1}, \cdots, z_{n-1} \in X$, for some $\rho>0$ and $\left.L>0\right\}$,

$W_{0}^{\prime \prime \prime}(\Delta, p,\|\cdot, \ldots, \cdot\|)=\left\{\left\langle a_{i j k}\right\rangle \in w^{\prime \prime \prime}: \lim _{l, m, n} \frac{1}{\operatorname{lmn}}\right.$

$\sum_{i=1}^{l} \sum_{j=1}^{m} \sum_{k=1}^{n}\left(\left\|\frac{\Delta a_{i j k}}{\rho}, z_{1}, \cdots, z_{n-1}\right\|\right)^{p_{i j k}}=0$,

for each $z_{1}, \cdots, z_{n-1} \in X$, for some $\left.\rho>0\right\}$, and

$W_{\infty}^{\prime \prime \prime}(M, \Delta, p,\|\cdot, \ldots, \cdot\|)$

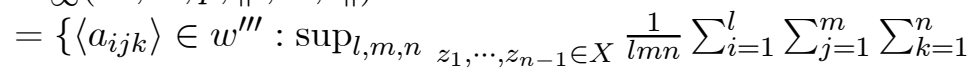

$\left(\left\|\frac{\Delta a_{i j k}}{\rho}, z_{1}, \cdots, z_{n-1}\right\|\right)^{p_{i j k}}<\infty$

for some $\rho>0\}$,

Theorem 3.1. Let $M$ be an Orlicz function and $p=\left(p_{i j k}\right)$ be bounded triple sequence of strictly positive real numbers. Then the classes of sequences $W^{\prime \prime \prime}(M, \Delta, p,\|\cdot, \ldots, \cdot\|), W_{0}^{\prime \prime \prime}(M, \Delta, p,\|\cdot, \ldots, \cdot\|)$ and

$W_{\infty}^{\prime \prime \prime}(M, \Delta, p,\|\cdot, \ldots, \cdot\|)$ are linear spaces over the field of real numbers $\mathbf{R}$.

Proof. Let $\left\langle a_{i j k}\right\rangle,\left\langle b_{i j k}\right\rangle \in W_{\infty}^{\prime \prime \prime}(M, \Delta, p,\|\cdot, \ldots, \cdot\|)$ and $\alpha, \beta \in \mathbf{R}$. Then there exist positive real numbers $\rho_{1}, \rho_{2}$ such that

$$
\sup _{\substack{l, m, n \\ z_{1}, \cdots, z_{n-1} \in X}} \frac{1}{\operatorname{lmn}} \sum_{i=1}^{l} \sum_{j=1}^{m} \sum_{k=1}^{n}\left[M\left(\left\|\frac{\Delta a_{i j k}}{\rho_{1}}, z_{1}, \cdots, z_{n-1}\right\|\right)\right]^{p_{i j k}}<\infty,
$$

for some $\rho_{1}>0$

and

$$
\sup _{\substack{l, m, n \\ z_{1}, \cdots, z_{n-1} \in X}} \frac{1}{l m n} \sum_{i=1}^{l} \sum_{j=1}^{m} \sum_{k=1}^{n}\left[M\left(\left\|\frac{\Delta a_{i j k}}{\rho_{2}}, z_{1}, \cdots, z_{n-1}\right\|\right)\right]^{p_{i j k}}<\infty,
$$

for some $\rho_{2}>0$. 
Let $\rho_{3}=\max \left(2|\alpha| \rho_{1}, 2|\beta| \rho_{2}\right)$, then since $\|\cdot, \ldots, \cdot\|$ is a $n$-norm on $X$ and $M$ is non-decreasing, convex and so by using inequality $(2.1)$, we have

$$
\begin{aligned}
& \sup _{l, m, n} z_{1}, \cdots, z_{n-1} \in X \\
& \frac{1}{l m n} \sum_{i=1}^{l} \sum_{j=1}^{m} \sum_{k=1}^{n}\left[M\left(\left\|\frac{\Delta\left[\alpha a_{i j k}+\beta b_{i j k}\right]}{\rho_{3}}, z_{1}, \cdots, z_{n-1}\right\|\right)\right]^{p_{i j k}} \\
& \leq \sup _{l, m, n} z_{z_{1}, \cdots, z_{n-1} \in X} \frac{1}{l m n} \sum_{i=1}^{l} \sum_{j=1}^{m} \sum_{k=1}^{n}\left[M \left(\left\|\frac{\Delta \alpha a_{i j k}}{\rho_{3}}, z_{1}, \cdots, z_{n-1}\right\|\right.\right. \\
& +\left\|\frac{\Delta \beta b_{i j k}}{\rho_{3}}, z_{1}, \cdots, z_{n-1}\right\|^{p_{i j k}} \\
& \leq K \sup _{l, m, n} z_{z_{1}, \cdots, z_{n-1} \in X} \frac{1}{l m n} \sum_{i=1}^{l} \sum_{j=1}^{m} \sum_{k=1}^{n} \frac{1}{2^{p_{i j k}}}\left[M\left(\left\|\frac{\Delta\left[a_{i j k}\right]}{\rho_{1}}, z_{1}, \cdots, z_{n-1}\right\|\right)\right]^{p_{i j k}} \\
& +K \sup _{l, m, n} z_{z_{1}, \cdots, z_{n-1} \in X} \frac{1}{l m n} \sum_{i=1}^{l} \sum_{j=1}^{m} \sum_{k=1}^{n} \frac{1}{2^{p_{i j k}}}\left[M\left(\left\|\frac{\Delta\left[b_{i j k}\right]}{\rho_{2}}, z_{1}, \cdots, z_{n-1}\right\|\right)\right]^{p_{i j k}} \\
& \leq K \sup _{l, m, n} z_{1}, \cdots, z_{n-1} \in X \frac{1}{l m n} \sum_{i=1}^{l} \sum_{j=1}^{m} \sum_{k=1}^{n}\left[M\left(\left\|\frac{\Delta\left[a_{i j k}\right]}{\rho_{1}}, z_{1}, \cdots, z_{n-1}\right\|\right)\right]^{p_{i j k}} \\
& +K \sup _{l, m, n} z_{z_{1}, \cdots, z_{n-1} \in X} \frac{1}{l m n} \sum_{i=1}^{l} \sum_{j=1}^{m} \sum_{k=1}^{n}\left[M\left(\left\|\frac{\Delta\left[b_{i j k}\right]}{\rho_{2}}, z_{1}, \cdots, z_{n-1}\right\|\right)\right]^{p_{i j k}} \\
& <\infty .
\end{aligned}
$$

Thus we have $\alpha\left\langle a_{i j k}\right\rangle+\beta\left\langle b_{i j k}\right\rangle \in W_{\infty}^{\prime \prime \prime}(M, \Delta, p,\|\cdot, \ldots, \cdot\|)$.

Hence $W_{\infty}^{\prime \prime \prime}(M, \Delta, p,\|\cdot, \ldots, \cdot\|)$ is a linear space. Similarly it can be shown that $W^{\prime \prime \prime}(M, \Delta, p,\|\cdot, \ldots, \cdot\|), W_{0}^{\prime \prime \prime}(M, \Delta, p,\|\cdot, \ldots, \cdot\|)$ are linear spaces over the field of reals $\mathbf{R}$.

Theorem 3.2. Let $M$ be an Orlicz function and $p=\left(p_{i j k}\right)$ be bounded triple sequence of strictly positive real numbers. The sequence spaces $W^{\prime \prime \prime}(M, \Delta, p,\|\cdot, \ldots, \cdot\|), W_{0}^{\prime \prime \prime}(M, \Delta, p,\|\cdot, \ldots, \cdot\|)$ and $W_{\infty}^{\prime \prime \prime}(M, \Delta, p,\|\cdot, \ldots, \cdot\|)$ are paranormed spaces, paranormed by

$$
\begin{aligned}
& g\left(\left\langle a_{i j k}\right\rangle\right)= \\
& \sup _{i}\left|a_{i 11}\right|+\sup _{j}\left|a_{1 j 1}\right|+\sup _{k}\left|a_{11 k}\right| \\
& +\inf \left\{\rho^{\frac{p_{i j k}}{H}}>0: \sup _{l, m, n} z_{1}, \cdots, z_{n-1} \in X\right. \\
&
\end{aligned}
$$

Where $H=\max (1, G), G=\sup _{i, j, k} p_{i j k}$.

Proof. Clearly $g(0)=0$ and $g\left(-\left\langle a_{i j k}\right\rangle\right)=g\left(\left\langle a_{i j k}\right\rangle\right)$.

Let $\left\langle a_{i j k}\right\rangle,\left\langle b_{i j k}\right\rangle \in W_{\infty}^{\prime \prime \prime}(M, \Delta, p,\|\cdot, \ldots, \cdot\|)$. The there exists some $\rho_{1}, \rho_{2}>0$ 
such that

$$
\sup _{\substack{l, m, n \\ z_{1}, \cdots, z_{n-1} \in X}}\left[M\left(\left\|\frac{\Delta a_{i j k}}{\rho_{1}}, z_{1}, \cdots, z_{n-1}\right\|\right)\right]^{\frac{p_{i j k}}{H}} \leq 1 .
$$

and

$$
\sup _{\substack{l, m, n \\ z_{1}, \cdots, z_{n-1} \in X}}\left[M\left(\left\|\frac{\Delta a_{i j k}}{\rho_{2}}, z_{1}, \cdots, z_{n-1}\right\|\right)\right]^{\frac{p_{i j k}}{H}} \leq 1 .
$$

Let $\rho=\rho_{1}+\rho_{2}$, then by using Minkowski's inequality, we have

$$
\begin{aligned}
& \sup _{l, m, n} z_{1}, \cdots, z_{n-1} \in X \\
& {\left[M\left(\left\|\frac{\Delta a_{i j k}+\Delta b_{i j k}}{\rho}, z_{1}, \cdots, z_{n-1}\right\|\right)\right]^{\frac{p_{i j k}}{H}}} \\
& \leq\left(\frac{\rho_{1}}{\rho_{1}+\rho_{2}}\right) \sup _{l, m, n} z_{z_{1}, \cdots, z_{n-1} \in X}\left[M\left(\left\|\frac{\Delta a_{i j k}}{\rho_{1}}, z_{1}, \cdots, z_{n-1}\right\|\right)\right]^{\frac{p_{i j k}}{H}} \\
& \quad+\left(\frac{\rho_{2}}{\rho_{1}+\rho_{2}}\right) \sup _{l, m, n} z_{z_{1}, \cdots, z_{n-1} \in X}\left[M\left(\left\|\frac{\Delta b_{i j k}}{\rho_{2}}, z_{1}, \cdots, z_{n-1}\right\|\right)\right]^{\frac{p_{i j k}}{H}} \leq 1 .
\end{aligned}
$$

Now 


$$
\begin{aligned}
& g\left(\left\langle a_{i j k}\right\rangle+\left\langle b_{i j k}\right\rangle\right) \\
& =\sup _{i}\left|a_{i 11}+b_{i 11}\right|+\sup _{j}\left|a_{1 j 1}+b_{1 j 1}\right|+\sup _{k}\left|a_{11 k}+b_{11 k}\right| \\
& +\inf \left\{\left(\rho_{1}+\rho_{2}\right)^{\frac{p_{i j k}}{H}}>0: \sup _{l, m, n} z_{1}, \cdots, z_{n-1} \in X\right. \\
& {\left[M\left(\left\|\frac{\Delta a_{i j k}+\Delta b_{i j k}}{\rho_{1}+\rho_{2}}, z_{1}, \cdots, z_{n-1}\right\|\right)\right]^{\frac{p_{i j k}}{H}} \leq 1} \\
& \leq \sup _{i}\left|a_{i 11}\right|+\sup _{i}\left|b_{i 11}\right|+\sup _{j}\left|a_{1 j 1}\right|+\sup _{j}\left|b_{1 j 1}\right|+\sup _{k}\left|a_{11 k}\right|+\sup _{k}\left|b_{11 k}\right| \\
& +\inf \left\{\left(\rho_{1}\right)^{\frac{p_{i j k}}{H}}>0: \sup _{l, m, n} z_{1}, \cdots, z_{n-1} \in X\right. \\
& {\left[M\left(\left\|\frac{\Delta a_{i j k}+\Delta b_{i j k}}{\rho_{1}}, z_{1}, \cdots, z_{n-1}\right\|\right)\right]^{\frac{p_{i j k}}{H}} \leq 1} \\
& +\inf \left\{\left(\rho_{2}\right)^{\frac{p_{i j k}}{H}}>0: \sup _{l, m, n} z_{1}, \cdots, z_{n-1} \in X\right. \\
& {\left[M\left(\left\|\frac{\Delta a_{i j k}+\Delta b_{i j k}}{\rho_{2}}, z_{1}, \cdots, z_{n-1}\right\|\right)\right]^{\frac{p_{i j k}}{H}} \leq 1} \\
& =g\left(\left\langle a_{i j k}\right\rangle\right)+g\left(\left\langle b_{i j k}\right\rangle\right) .
\end{aligned}
$$

Let $\lambda \in \mathbf{C}$ then the continuity of the product follows from the following inequality

$$
\begin{aligned}
& g\left(\lambda\left\langle a_{i j k}\right\rangle\right) \\
& =\sup _{i}\left|\lambda a_{i 11}\right|+\sup _{j}\left|\lambda a_{1 j 1}\right|+\sup _{k}\left|\lambda a_{11 k}\right| \\
& +\inf \left\{(\rho)^{\frac{p_{i j k}}{H}}>0: \sup _{l, m, n} z_{1}, \cdots, z_{n-1} \in X\left[M\left(\left\|\frac{\Delta \lambda a_{i j k}}{\rho}, z_{1}, \cdots, z_{n-1}\right\|\right)\right]^{\frac{p_{i j k}}{H}} \leq 1\right\} \\
& =|\lambda|\left(\sup _{i}\left|a_{i 11}\right|+\sup _{j}\left|a_{1 j 1}\right|+\sup _{k}\left|a_{11 k}\right|\right)
\end{aligned}
$$

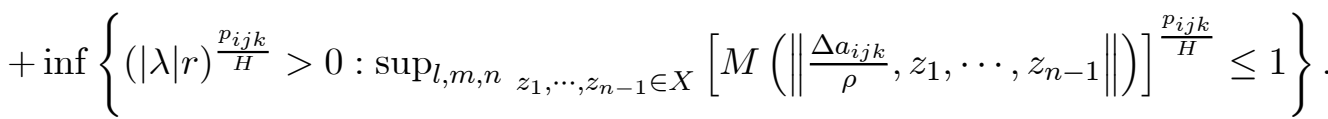

Where $\frac{1}{r}=\frac{|\lambda|}{\rho}$.

This completes the proof of the theorem. 
Theorem 3.3. Let $M$ be an Orlicz function and $p=\left(p_{i j k}\right)$ be bounded triple sequence of strictly positive real numbers. The sequence spaces $W^{\prime \prime \prime}(M, \Delta, p,\|\cdot, \ldots, \cdot\|), W_{0}^{\prime \prime \prime}(M, \Delta, p,\|\cdot, \ldots, \cdot\|)$ and $W_{\infty}^{\prime \prime \prime}(M, \Delta, p,\|\cdot, \ldots, \cdot\|)$ are complete paranormed spaces, under the paranorm defined by $g$.

Proof. Let $\left\langle a_{i j k}^{s}\right\rangle$ be a Cauchy sequence in $W_{\infty}^{\prime \prime \prime}(M, \Delta, p,\|\cdot, \ldots, \cdot\|)$. Then $g\left(\left\langle a_{i j k}^{s}-a_{i j k}^{t}\right) \rightarrow 0\right.$ as $s, t \rightarrow \infty$. For a given $\epsilon>0$, choose $r>0$ and $x_{0}>0$ be such that $\frac{\epsilon}{r x_{0}}>0$ and $M\left(\frac{r x_{0}}{2}\right) \geq 1$. Now $g\left(\left\langle a_{i j k}^{s}-a_{i j k}^{t}\right\rangle\right) \rightarrow 0$ as $s, t \rightarrow \infty$ implies that there exists $m_{0} \in \mathbf{N}$ such that

$$
g\left(\left\langle a_{i j k}^{s}-a_{i j k}^{t}\right\rangle\right)<\frac{\epsilon}{r x_{0}} \text { for all } s, t \geq m_{0} .
$$

Thus, we have

$$
\begin{aligned}
& \sup _{i} \\
& \left|a_{i 11}^{s}-a_{i 11}^{t}\right|+\sup _{j}\left|a_{1 j 1}^{s}-a_{1 j 1}^{t}\right|+\sup _{k}\left|a_{11 k}^{s}-a_{11 k}^{t}\right| \\
& +\inf \left\{(\rho)^{\frac{p_{i j k}}{H}}>0: \sup _{l, m, n} z_{1}, \cdots, z_{n-1} \in X\right. \\
& {\left[M\left(\left\|\frac{\Delta a_{i j k}^{s}-\Delta a_{i j k}^{t}}{\rho}, z_{1}, \cdots, z_{n-1}\right\|\right)\right]^{\frac{p_{i j k}}{H}} \leq 1} \\
& <\frac{\epsilon}{r x_{0}} .
\end{aligned}
$$

This shows that $\left\langle a_{i 11}^{s}\right\rangle,\left\langle a_{1 j 1}^{s}\right\rangle$ and $\left\langle a_{11 k}^{s}\right\rangle$ are Cauchy sequences of real numbers. As the set of real numbers is complete so there exists real numbers $a_{i 11}, a_{1 j 1}, a_{11 k}$ such that

$$
\lim _{s \rightarrow \infty} a_{i 11}^{s}=a_{i 11} \quad, \quad \lim _{s \rightarrow \infty} a_{1 j 1}^{s}=a_{1 j 1} \quad, \quad \lim _{s \rightarrow \infty} a_{11 k}^{s}=a_{11 k} .
$$

Then from (3.1) we have

$$
\begin{gathered}
\left(\left\|\frac{\Delta a_{i j k}^{s}-\Delta a_{i j k}^{t}}{\rho}, z_{1}, \cdots, z_{n-1}\right\|\right) \leq 1 \\
\Rightarrow \sup _{i, j, k}\left[M\left(\left\|\frac{\Delta a_{i j k}^{s}-\Delta a_{i j k}^{t}}{\rho}, z_{1}, \cdots, z_{n-1}\right\|\right)\right] \leq 1 \leq M\left(\frac{r x_{0}}{2}\right) \\
\Rightarrow \frac{\left\|\left(\Delta a_{i j k}^{s}-\Delta a_{i j k}^{t}\right), z_{1}, \cdots, z_{n-1}\right\|}{g\left(\left\langle a_{i j k}^{s}-a_{i j k}^{t}\right\rangle\right)} \leq \frac{r x_{0}}{2} \\
\Rightarrow\left\|\left(\Delta a_{i j k}^{s}-\Delta a_{i j k}^{t}\right), z_{1}, \cdots, z_{n-1}\right\|<\frac{r x_{0}}{2} \cdot \frac{\epsilon}{r x_{0}}=\frac{\epsilon}{2} .
\end{gathered}
$$


This implies that $\left\langle\Delta a_{i j k}^{s}\right\rangle$ is a Cauchy sequence of real numbers. Let $\lim _{s \rightarrow \infty} \Delta a_{i j k}^{s}=y_{i j k}$ for all $i, j, k \in \mathbf{N}$. Now

$$
\begin{aligned}
& \Delta a_{111}^{s}=a_{111}^{s}-a_{112}^{s}-a_{121}^{s}-a_{211}^{s}+a_{122}^{s}+a_{212}^{s}+a_{221}^{s}-a_{222}^{s} . \\
& \lim _{s \rightarrow \infty} a_{222}^{s} \\
& \text { So } \quad=\lim _{s \rightarrow \infty}\left[a_{111}^{s}-a_{112}^{s}-a_{121}^{s}-a_{211}^{s}+a_{122}^{s}+a_{212}^{s}+a_{221}^{s}-\Delta a_{111}^{s}\right] \\
&=a_{111}-a_{112}-a_{121}-a_{211}+a_{122}+a_{212}+a_{221}-y_{111} .
\end{aligned}
$$

Thus $\lim _{s \rightarrow \infty} a_{222}^{s}$ exists. Proceeding in this way we conclude that $\lim _{s \rightarrow \infty} a_{i j k}^{s}$ exists. Using the continuity of $M$, we have

$$
\lim _{t \rightarrow \infty} \sup _{\substack{i, j, k \\ z_{1}, \cdots, z_{n-1}}}\left[M\left(\left\|\frac{\Delta a_{i j k}^{s}-\Delta a_{i j k}^{t}}{\rho}, z_{1}, \cdots, z_{n-1}\right\|\right)\right] \leq 1 .
$$

Let $s \geq m_{0}$, then taking infimum of such $\rho$ 's we have $g\left(\left\langle a_{i j k}^{s}-a_{i j k}^{t}\right\rangle\right)<$ $\epsilon$. Thus $\left\langle a_{i j k}^{s}-a_{i j k}\right\rangle \in W_{\infty}^{\prime \prime \prime}(M, \Delta, p,\|\cdot, \ldots, \cdot\|)$. By linearity of the space $W_{\infty}^{\prime \prime \prime}(M, \Delta, p,\|\cdot, \ldots, \cdot\|)$ we have $\left\langle a_{i j k}\right\rangle \in W_{\infty}^{\prime \prime \prime}(M, \Delta, p,\|\cdot, \ldots, \cdot\|)$. Hence $W_{\infty}^{\prime \prime \prime}(M, \Delta, p,\|\cdot, \ldots, \cdot\|)$ is complete.

Theorem 3.4. Let $M$ be an Orlicz function and $p=\left(p_{i j k}\right)$ be bounded triple sequence of strictly positive real numbers. Then

(i) $W^{\prime \prime \prime}(M, \Delta, p,\|\cdot, \ldots, \cdot\|) \subset W_{\infty}^{\prime \prime \prime}(M, \Delta, p,\|\cdot, \ldots, \cdot\|)$

(ii) $W_{0}^{\prime \prime \prime}(M, \Delta, p,\|\cdot, \ldots, \cdot\|) \subset W_{\infty}^{\prime \prime \prime}(M, \Delta, p,\|\cdot, \ldots, \cdot\|)$

Proof. The proof is easy so we omit it.

Theorem 3.5. Let $M$ be an Orlicz function and $p=\left(p_{i j k}\right)$ be bounded triple sequence of strictly positive real numbers. Then the following relation holds

(i) If $0<\inf p_{i j k} \leq p_{i j k}<1$, then $W^{\prime \prime \prime}(M, \Delta, p,\|\cdot, \ldots, \cdot\|) \subseteq W^{\prime \prime \prime}(M, \Delta,\|\cdot, \ldots, \cdot\|)$

(ii) If $0<p_{i j k} \leq \sup p_{i j k}<\infty$, then $W^{\prime \prime \prime}(M, \Delta,\|\cdot, \ldots, \cdot\|) \subseteq W^{\prime \prime \prime}(M, \Delta, p,\|\cdot, \ldots, \cdot\|)$ 
Proof. (i) Let $\left\langle a_{i j k}\right\rangle \in W^{\prime \prime \prime}(M, \Delta, p,\|\cdot, \ldots, \cdot\|)$; since $0<\inf \left\{p_{i j k}\right\} \leq$ $p_{i j k}<1$, we have

$$
\begin{aligned}
& \sup _{l, m, n_{z_{1}}, \cdots, z_{n-1} \in X} \\
& \frac{1}{\operatorname{lmn}} \sum_{i=1}^{l} \sum_{j=1}^{m} \sum_{k=1}^{n}\left[M\left(\left\|\frac{\Delta a_{i j k}}{\rho}, z_{1}, \cdots, z_{n-1}\right\|\right)\right] \\
& \leq \sup _{l, m, n_{z_{1}, \cdots, z_{n-1} \in X}} \frac{1}{\operatorname{lmn}} \sum_{i=1}^{l} \sum_{j=1}^{m} \sum_{k=1}^{n} \\
& {\left[M\left(\left\|\frac{\Delta a_{i j k}}{\rho}, z_{1}, \cdots, z_{n-1}\right\|\right)\right]^{p_{i j k}}}
\end{aligned}
$$

and hence $\left\langle a_{i j k}\right\rangle \in W^{\prime \prime \prime}(M, \Delta,\|\cdot, \ldots, \cdot\|)$.

(ii) Let $p_{i j k}>1$ for each $(i j k)$ and $\sup _{i, j, k} p_{i j k}<\infty$. Let $\left\langle a_{i j k}\right\rangle \in W^{\prime \prime \prime}(M, \Delta,\|\cdot, \ldots, \cdot\|)$.

Then, for each $0, \epsilon<1$, there exists a positive integer $\mathbf{N}$ such that

$$
\sup _{\substack{l, m, n \\ z_{1}, \cdots, z_{n-1} \in X}} \frac{1}{\operatorname{lmn}} \sum_{i=1}^{l} \sum_{j=1}^{m} \sum_{k=1}^{n}\left[M\left(\left\|\frac{\Delta a_{i j k}}{\rho}, z_{1}, \cdots, z_{n-1}\right\|\right)\right] \leq \epsilon<1
$$

for all $m, n \geq \mathbf{N}$. Since

$$
\begin{aligned}
& \sup _{l, m, n} z_{1}, \cdots, z_{n-1} \in X \\
& \frac{1}{l m n} \sum_{i=1}^{l} \sum_{j=1}^{m} \sum_{k=1}^{n}\left[M\left(\left\|\frac{\Delta a_{i j k}}{\rho}, z_{1}, \cdots, z_{n-1}\right\|\right)\right]^{p_{i j k}} \\
& \leq \sup _{l, m, n} z_{1}, \cdots, z_{n-1} \in X \\
& \frac{1}{l m n} \sum_{i=1}^{l} \sum_{j=1}^{m} \sum_{k=1}^{n}\left[M\left(\left\|\frac{\Delta a_{i j k}}{\rho}, z_{1}, \cdots, z_{n-1}\right\|\right)\right]
\end{aligned}
$$

Hence $\left\langle a_{i j k}\right\rangle \in W^{\prime \prime \prime}(M, \Delta, p,\|\cdot, \ldots, \cdot\|)$ which completes the proof.

Theorem 3.6. Let $M_{1}$ and $M_{2}$ be Orlicz functions, then we have $W_{\infty}^{\prime \prime \prime}\left(M_{1}, \Delta, p,\|\cdot, \ldots, \cdot\|\right)$

$$
\begin{aligned}
& \cap W_{\infty}^{\prime \prime \prime}\left(M_{2}, \Delta, p,\|\cdot, \ldots, \cdot\|\right) \\
& \subseteq W_{\infty}^{\prime \prime \prime}\left(M_{1}+M_{2}, \Delta, p,\|\cdot, \ldots, \cdot\|\right)
\end{aligned}
$$

Proof. $\quad$ Let $\left\langle a_{i j k}\right\rangle \in W_{\infty}^{\prime \prime \prime}\left(M_{1}, \Delta, p,\|\cdot, \ldots, \cdot\|\right) \cap W_{\infty}^{\prime \prime \prime}\left(M_{2}, \Delta, p,\|\cdot, \ldots, \cdot\|\right)$.

Then

$$
\sup _{\substack{l, m, n \\ z_{1}, \cdots, z_{n-1} \in X}} \frac{1}{l m n} \sum_{i=1}^{l} \sum_{j=1}^{m} \sum_{k=1}^{n}\left[M_{1}\left(\left\|\frac{\Delta a_{i j k}}{\rho_{1}}, z_{1}, \cdots, z_{n-1}\right\|\right)\right]^{p_{i j k}}<\infty, \quad \text { for some } \rho_{1}>0
$$

and 


$$
\sup _{\substack{l, m, n \\ z_{1}, \cdots, z_{n-1} \in X}} \frac{1}{\operatorname{lmn}} \sum_{i=1}^{l} \sum_{j=1}^{m} \sum_{k=1}^{n}\left[M_{2}\left(\left\|\frac{\Delta a_{i j k}}{\rho_{2}}, z_{1}, \cdots, z_{n-1}\right\|\right)\right]^{p_{i j k}}<\infty, \quad \text { for some } \rho_{1}>0
$$

Let $\rho=\max \left\{\rho_{1}, \rho_{2}\right\}$. The result follows from the inequality

$$
\begin{aligned}
& \sum_{i=1}^{l} \sum_{j=1}^{m} \sum_{k=1}^{n} \\
& {\left[\left(M_{1}+M_{2}\right)\left(\left\|\frac{\Delta a_{i j k}}{\rho}, z_{1}, \cdots, z_{n-1}\right\|\right)\right]^{p_{i j k}}} \\
& =\sum_{i=1}^{l} \sum_{j=1}^{m} \sum_{k=1}^{n}\left[M_{1}\left(\left\|\frac{\Delta a_{i j k}}{\rho}, z_{1}, \cdots, z_{n-1}\right\|\right)+M_{2}\left(\left\|\frac{\Delta a_{i j k}}{\rho}, z_{1}, \cdots, z_{n-1}\right\|\right)\right]^{p_{i j k}} \\
& \leq K \sum_{i=1}^{l} \sum_{j=1}^{m} \sum_{k=1}^{n}\left[M_{1}\left(\left\|\frac{\Delta a_{i j k}}{\rho}, z_{1}, \cdots, z_{n-1}\right\|\right)\right]^{p_{i j k}} \\
& \quad+K \sum_{i=1}^{l} \sum_{j=1}^{m} \sum_{k=1}^{n}\left[M_{1}\left(\left\|\frac{\Delta a_{i j k}}{\rho}, z_{1}, \cdots, z_{n-1}\right\|\right)\right]^{p_{i j k}}
\end{aligned}
$$

Theorem 3.7. The sequence space $W_{\infty}^{\prime \prime \prime}(M, \Delta, p,\|\cdot, \ldots, \cdot\|)$ is solid.

Proof. $\quad$ Let $\left\langle a_{i j k}\right\rangle \in W_{\infty}^{\prime \prime \prime}(M, \Delta, p,\|\cdot, \ldots, \cdot\|)$, that is

$$
\sup _{\substack{l, m, n \\ z_{1}, \cdots, z_{n-1} \in X}} \frac{1}{\operatorname{lmn}} \sum_{i=1}^{l} \sum_{j=1}^{m} \sum_{k=1}^{n}\left[M\left(\left\|\frac{\Delta a_{i j k}}{\rho}, z_{1}, \cdots, z_{n-1}\right\|\right)\right]^{p_{i j k}}<\infty
$$

Let $\left(\alpha_{i j k}\right)$ be a triple sequence of scalars such that $\left|\alpha_{i j k}\right| \leq 1$ for all $i, j, k \in \mathbf{N}$.

Then we get

$$
\begin{aligned}
& \sup _{l, m, n} n_{z_{1}, \cdots, z_{n-1} \in X} \frac{1}{\operatorname{lmn}} \\
& \sum_{i=1}^{l} \sum_{j=1}^{m} \sum_{k=1}^{n}\left[M\left(\left\|\frac{\Delta \alpha_{i j k} a_{i j k}}{\rho}, z_{1}, \cdots, z_{n-1}\right\|\right)\right]^{p_{i j k}}
\end{aligned}
$$

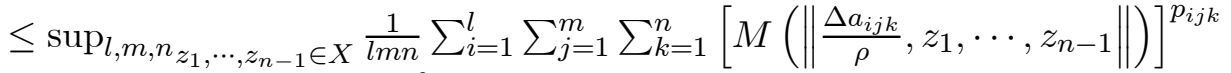
and this completes the proof.

Theorem 3.8. The sequence space $W_{\infty}^{\prime \prime \prime}(M, \Delta, p,\|\cdot, \ldots, \cdot\|)$ is monotone. 
Proof. The result is obvious.

\section{Acknowledgement :}

The authors are thankful to the reviewers for their valuable suggestions and comments which improved the presentation of the paper.

\section{References}

[1] A. Alotaibi, M. Mursaleen and S. K. Sharma, Double sequence spaces over $n$-normed spaces defined by a sequence of orlicz functions, Jou. Ineq. Appl., 216, 2014:216, (2014).

[2] A. J. Datta, A. Esi and B.C. Tripathy, Statistically convergent triple sequence space defined by orlicz function, Jou. Math. Anal., 4, pp. 16-22, (2013).

[3] S. Debnath, B.C. Sarma Das, Some generalized triple sequence spaces of real numbers, Jou. Nonl. Anal. and Opt., 6, pp. 71-79, (2015).

[4] H. Dutta, An application of lacunary summability method to $n$-norm, Int. Jou. Appl. Math. Sat., 15 (D09), pp. 89-97, (2009).

[5] H. Dutta, On sequence spaces with elements in a sequence of real linear n-normed spaces, Appl. Math. Lett., 33(9), pp. 1109-113, (2010).

[6] H. Dutta, On some $n$-normed linear space valued difference sequence, Jou. Franklin Inst., 348, pp. 2876-2883, (2011).

[7] A. Esi, On some triple almost lacunary sequence spaces defined by orlicz functions, Research and Reviews: Discrete Mathematical structures 1, pp. 16-25, (2014).

[8] A. Esi, M.N. Catalbas, Almost convergence of triple sequences, G. Jou. Math. Anal., 2, pp. 6-10, (2014).

[9] A. Esi, E. Savas, On lacunary statically convergent triple sequences in probabilistic normed space, Appl. Math. Inf. Sci., 9, pp. 2529-2534, (2015).

[10] M. Et, R. Colak, On generalized difference sequence spaces, Soochow Jou. Math., 21, 4, pp. 377-386, (1995). 
[11] S. Gähler, Linear 2-normietre rume, Math. Nachr., 28, pp. 1-43, (1965).

[12] H. Gunawan, On $n$-inner product, $n$-norms, and the cauchySchwartz Inequality, Sci. Math. Jap., 5, pp. 47-54, (2001).

[13] H. Gunawan, The space of $p$-summable sequence and its natural $n$-norm, Bull. Austral. Math. Soc., 64, pp. 137-147, (2001).

[14] H. Gunawan, M. Mashadi, On n-normed spaces, Int. Jou. Math. Sci., 27, pp. 631-639, (2001).

[15] T. Jalal, Some new I-convergent sequence spaces defined by using a sequence of modulus functions in $n$-normed spaces, Int. Jou. Math. Archive, 5 (9), pp. 202-209, (2014).

[16] T. Jalal, On generalized A-difference sequence spaces defined by ideal convergence on a real $n$-normed space through lacunary sequences, Bull. Cal. Math. Soc., 106 (6), pp. 415-426 (2014).

[17] T. Jalal, Some new I-lacunary generalized difference sequence spaces defined in $n$-normed spaces, Springer Proc. Math. Stat., 171, pp. 249258, (2016).

[18] P.K. Kamthan, M. Gupta, Sequence spaces and series 1st Edn., M. Dekker, New York, ISBN 0-8247-1224-2, pp. 298-300, (1981).

[19] H. Kizmaz, On certain sequence spaces, Canad. Math. Bull. 24 (1), pp. 169-176, (1981).

[20] J. Lindenstrauss, L. Tzafriri, On orlicz sequence spaces, Israel J. Math., 10, pp. 379-390, (1971).

[21] A. Misiak, n-Inner product spaces, Math. Nachr., 140, pp. 299-319, (1989).

[22] M. Mursaleen, K. Raj and S. K. Sharma, Some spaces of differences and lacunary statistical convergence in $n$-normed space defined by sequence of orlicz functions, Miskolc Mathematical Notes, 16(1), pp. 283-304, (2015).

[23] M. Mursaleen, S. K. Sharma and A. Kilicman, Sequence spaces defined by musielak-orlicz function over $n$-normed spaces, Abs. and Appl. Anal. Vol. 2013, ID 364743, (2013). 
[24] J. Musielak, Orlicz spaces and modular spaces, 1st Edn., Springer, New York, ISBN 3-5401-2706-2, pp. 33-112, (1983).

[25] K. Raj, A.K. Sharma and S.K. Sharma, A sequence space defined by musielak-orlicz functions, Int. Jou. Pure Appl. Math., 67, pp. 472-484, (2011).

[26] K. Raj, A.K. Sharma and S.K. Sharma, Some new sequence spaces defined by a sequence of modulus functions in $n$-normed spaces, Int. Jou. Math. Sci. Engg. Appl., 5, pp. 395-403, (2011).

[27] A. Sahiner, M. Gurdal and F.K. Duden , Triple sequences and their statistical convergence, Selcuk Jou. Appl. Math., 8, pp. 49-55, (2007).

[28] A. Sahiner, B.C. Tripathy , Some I related properties of triple sequences, Selcuk Jou. Appl. Math., 9, pp. 9-18, (2008).

[29] E. Savas, On some new sequence spaces in 2-normed spaces using ideal convergence and an orlicz function, Jou. Ineq. Appl., Vol. 2010, ID 482392, (2010).

[30] A. Wilansky, Modern methods in topological vector spaces, McGrawHill, ISBN-0-07-070180-6, (1978).

\section{Tanweer Jalal}

Department of Mathematics, National Institute of Technology,

Hazratbal, Srinagar-190006, Jammu and Kashmir, India

e-mail tjalal@nitsri.net

and

\section{Ishfaq Ahmad Malik}

Department of Mathematics, National Institute of Technology,

Hazratbal, Srinagar-190006, Jammu and Kashmir, India

e-mail : ishfaq_2phd15@nitsri.net 